\title{
Adaptación local del Cuestionario Revisado de Personalidad de Eysenck
}

\author{
(Versión abreviada) \\ Mario Squillace******, Jimena Picón Janeiro ***** \& Vanina Schmidt ***** \\ *Universidad de Buenos Aires, Argentina. **Pontificia Universidad Católica Argentina. \\ ***Universidad del Salvador, Argentina. ****Consejo Nacional de Investigaciones Científicas y Técnicas, \\ Argentina.
}

\begin{abstract}
Resumen. Se presenta la adaptación del Cuestionario Revisado de Personalidad de Eysenck (versión abreviada). El mismo fue administrado en la Ciudad de Buenos Aires $(\mathrm{n}=811)$. Se realizó un análisis factorial exploratorio a través del análisis de componentes principales para extraer la cantidad de factores, aplicando criterios estadísticos exigentes. Se extrajeron los tres factores de personalidad postulados por el modelo Psicoticismo-Extroversión-Neuroticismo (PEN) y un factor de sinceridad. Se realizaron los mismos análisis por separado para las submuestras de hombres y mujeres. Para estimar la confiabilidad del instrumento se aplicó alfa de Cronbach, observándose índices de consistencia interna adecuados para cada subescala. De este modo, se ha obtenido la versión local del EPQ-RA que consta de 42 ítems distribuidos en tres factores robustos que se corresponden con las tres dimensiones de personalidad más prolíficas de la historia de la Psicología, y un factor de sinceridad igualmente válido y consistente desde el punto de vista psicométrico.
\end{abstract}

Palabras clave: Cuestionario - Personalidad - Adaptación - Modelo PEN

\section{Local Adaptation of Eysenck Personality Questionnarie (Short Form)}

\begin{abstract}
A local adaptation of the Eysenck Personality Questionnaire Revised (abbreviated version) is presented. It was applied at Buenos Aires city $(\mathrm{n}=811)$. An exploratory factor analysis was applied through principal components technique. To extract factors, rigorous statistics criteria were followed. Three factors were extracted which represent the three main personality dimensions hypothesized by the Psychoticism-Extroversion-Neuroticism (PEN) Model and one for the sincerity assessment. The same analyses were conducted for women and men samples. To evaluate the instrument reliability Cronbach alpha was applied and adequate internal consistency coefficients were observed. Thus, the local version of EPQRA is obtained, with 42 items distributed through four factors: three of them which represent the more prolific personality dimension in Psychology history, and the last one, is a "sincerity factor" equally valid and reliable.
\end{abstract}

Key Words: Questionnaire - Personality - Adaptation - PEN model

\section{Introducción}

El modelo de personalidad de Eysenck tiene más de 50 años y es posiblemente uno de los más robustos que la Psicología ha ofrecido hasta nuestros días. Eysenck $(1987,1990)$ se basó en un amplio espectro de ideas y evidencias. Por ejemplo, retomó las viejas observaciones realizadas por la teoría hipocrático-galénica de los humores, consideraciones filosóficas como las de Kant y Jung, y los aportes actuales de la psicología experimental y las neurociencias. Su vasto trabajo ha forjado un modelo psicobiológico de la personalidad que constituye en la actualidad un verdadero paradigma de trabajo, el cual debe considerarse obligadamente en el debate de la psicología científica moderna. 
Eysenck postulaba que el elemento central que permite integrar las causas proximales y distales de la conducta es la personalidad. La evidencia empírica acumulada a través de los años brinda un fuerte apoyo a sus originales y controvertidas ideas. Las últimas contribuciones que se han hecho a su modelo desde la biología y genética de la conducta lo han enriquecido logrando que el mismo cumpla con requisitos metodológicos y cualidades científicas que sólo unas pocas teorías han logrado alcanzar. Aunque posteriormente muchos científicos han discutido y modificado las hipótesis originales de Eysenck (Squillace, 2009; Squillace, Martin, Gianotti, Pedrón, \& Yorio, 2008), sus propuestas aún siguen siendo objeto de estudio.

Eysenck creó diferentes instrumentos para medir sus constructos teóricos. Inicialmente comenzó sus investigaciones utilizando el Maudsley Medical Questionnaire (MMQ; Eysenck, 1952), que luego fue revisado y modificado dando lugar al Maudsley Personality Inventory (MPI; Eysenck, 1959) que permitía medir Neuroticismo (N) y Extroversión (E). Unos años más tarde, Eysenck amplió el conocimiento sobre estos dos primeros grandes factores de la personalidad y diseñó un nuevo inventario para poder medirlos adecuadamente, que incluía adicionalmente una escala de sinceridad: el Eysenck Personality Inventory (EPI; Eysenck \& Eysenck, 1964). Un hallazgo esperado fue la asociación de los trastornos neuróticos con una mayor intensidad de la característica Neuroticismo. Sin embargo, lo más trascendente fue hallar que la cualidad del trastorno neurótico se encontraba influida por el grado de Introversión-Extroversión que poseyera la persona. De este modo, individuos con alto Neuroticismo e Introvertidos tendían al desarrollo de psicastenia, mientras que aquellos con mayores niveles de Extroversión tendían a la histeria (Eysenck, 1987). La polaridad Introversión-Extroversión (I-E) podía modular la formación de las patologías neuróticas interactuando con el Neuroticismo.

Análogamente, Kretschmer propuso un continuo entre la esquizofrenia en un polo (esquizotimia extrema) y la enfermedad maníaco-depresiva en el otro (ciclotimia extrema). Esto hizo pensar a Eysenck en la existencia de una tercera dimensión de personalidad: P (que explicaría la disposición o intensidad del trastorno psicótico). Algunos autores (MacKinnon, 1944; citado en Gutiérrez-Maldonado, 1999) relacionaron la esquizofrenia con Introversión y la psicosis maníaco-depresiva con Extroversión. En consecuencia, el modelo incluía tres dimensiones: dos ( $\mathrm{N}$ y $\mathrm{P}$ ) referidas a la disposición a sufrir trastornos neuróticos o psicóticos, y una tercera (E) que explicaría el tipo específico de trastorno neurótico o psicótico (Schmidt, 
Firpo, Vion, De Costa Oliván, Casella, Cuenya, et al., 2009). Para poder observar este nuevo constructo Eysenck modifica el EPI y desarrolla el Eysenck Personality Questionnaire (EPQ; Eysenck \& Eysenck, 1975; Eysenck, \& Eysenck, 1983), inventario del cual se realizan varias versiones, siendo la última el Eysenck Personality Questionnaire Revised (EPQ-R; Eysenck \& Eysenck, 2001). La inclusión de este nuevo factor de personalidad generó reajustes factoriales en la nueva teoría obligando, por ejemplo, a ubicar a la impulsividad (característica que hasta ese momento se teorizaba como parte de la Extroversión) como asociada al Psicoticismo (Squillace, Picón Janeiro, \& Schmidt, 2011). Finalmente, las cuatro dimensiones medidas por el EPQ-R son Extroversión, Neuroticismo, Psicoticismo y Sinceridad. Esta última escala corresponde a una medida de deseabilidad social que permite estimar qué tan confiables son las respuestas del individuo al contestar el inventario, aunque también puede estar evaluando algún rasgo de conformidad social del sujeto.

La escala Extroversión mide la necesidad del individuo de variar y buscar estimulación atribuida a bajos niveles de actividad cortical. La exigencia por estimularse y aumentar así su activación cortical explicaría la mayor disposición del extravertido al contacto social, al riesgo, a la búsqueda de nuevas sensaciones y a la acción general. Los introvertidos por el contrario pueden alcanzar niveles óptimos de activación cortical con menor estimulación, por lo que sus conductas estarían más orientadas hacia situaciones que impliquen poca activación, siendo más reservados en las situaciones sociales, más rutinarios y menos propensos al riesgo. Parecería no existir una relación lineal entre ejecución y arousal, sino que se trataría de una curva en forma de U invertida. Además la concepción de arousal psicobiológico general ha sufrido modificaciones (Stelmack, 1990). En la actualidad, el sistema retículo-cortical se considera únicamente como uno de los varios sistemas de arousal. Es probable que incluyan el sistema límbico de arousal, el sistema de la monoamino-oxidasa (MAO), el sistema difuso tálamo-cortical y el sistema pituitarioadrenocortical (Pelechano-Barberá, 2000). Esta aparente diversidad no excluye que los sistemas operen en forma relativamente unitaria (Eysenck, 1990). De cualquier manera, los estudios que se han realizado hasta el momento suponen un aceptable apoyo a la teoría del arousal (Eysenck, 1990; Wilson, 1990; Zuckerman, 1992), si bien no es suficiente para explicar la dimensión E.

Eysenck (1987) destaca dos rasgos centrales en la dimensión Extroversión: sociabilidad y actividad. Un individuo Extrovertido es sociable, vivaz, activo, asertivo, 
buscador de sensaciones socializadas, despreocupado, dominante, espontáneo y aventurero (Eysenck, 1990). Estas son las características que se contemplan en la escala Extroversión de los inventarios EPQ.

La escala Neuroticismo evalúa una dimensión que involucra respuestas emocionales intensas y estrés frente estímulos que perturban la homeostasis del individuo. Implica una predisposición frente a lo que clásicamente se conoce como trastornos neuróticos, tanto trastornos de ansiedad como del estado de ánimo. Un individuo con alto Neuroticismo puede padecer ansiedad, se deprime más fácilmente, tiende a la tensión emocional, a respuestas irracionales, a la inhibición, y a sentimientos de desvalorización y culpa (Eysenck, 1990).

La escala Psicoticismo valora el rasgo dureza emocional, que es conceptualizado como un continuo entre la normalidad, la psicopatía y la psicosis. Un individuo con alto Psicoticismo tiende a ser agresivo, hostil, frío, egocéntrico, impersonal, impulsivo, antisocial, creativo, rígido y poco empático. Por el contrario, un individuo con bajo Psicoticismo tiende a ser altruista, empático, responsable, socializado y convencional (Eysenck, 1990).

Extroversión, Neuroticismo y Psicoticismo (o Dureza) son para Eysenck las dimensiones básicas de la personalidad. Las personas pueden ser descritas en función del grado que exhiben en estos tres factores y, así, pueden ser ubicadas en algún punto del espacio tridimensional.

Las diferencias individuales en Extraversión, Neuroticismo y Psicoticismo son importantes en la vida diaria. Las relaciones entre personalidad y el comportamiento en la vida real han sido exploradas con el EPQ-R.

Las predicciones respecto de las diferencias comportamentales entre introvertidos y extravertidos han sido contrastadas tanto cuando se indaga el rendimiento en tareas de laboratorio, como cuando se estudian conductas a nivel social, académico y laboral (Eysenck \& Eysenck, 1994, 2001). La teoría de reactivación de H. J. Eysenck (1967) ha generado una multitud de predicciones, la mayoría de las cuales han encontrado algún apoyo experimental. A favor de la idea de un mayor nivel de activación, se hallaron correlaciones positivas entre Extraversión y la concentración de cortisol y colesterol en sangre (LeBlanc \& Ducharme, 2005). Precisamente la idea de que el nivel superior de los introvertidos frente a los extravertidos les lleva a estos últimos a necesitar aumentar la estimulación ha resultado de suma importancia para poder desentrañar una multiplicidad de experiencias de la vida cotidiana y de resultados sobre la ejecución de la tarea que tiene los altos Extraversión 
(Pelechano-Bareberá, 2000; Wilson, 1990; Zuckerman, 1992).

Asimismo, abundan los estudios con la dimensión Neuroticismo. En un estudio en el que se utilizó un electroencefalograma, se halló una mayor actividad relativa en el lóbulo parietal y temporal posterior del hemisferio derecho en los sujetos con valores elevados en Neuroticismo (Schmidtke \& Heller, 2004). En algunos estudios (por ejemplo, Brody \& Ehrlichman, 2000) se ha encontrado que sujetos con altos niveles en Neuroticismo muestran mayores indicadores psicofisiológicos de ansiedad frente a distintos estresores, pero la vinculación entre Neuroticismo y medidas psicofisiológicas de ansiedad y estrés es parcial, tal como los mimos autores señalan y el propio Eysenck (2001) reconoce.

Dentro del espacio bidimensional de la personalidad, se ha estudiado la combinación de Extraversión y Neuroticismo y su impacto a nivel psicopatológico. Se encontró que un alto puntaje en histeria se asociaba tanto a introversión con alto Neuroticismo como extraversión con alto Neuroticismo, y la criminalidad y psicopatía con la Extraversión neurótica. Al incluir la dimensión Psicoticismo, se complejizan los estudios y las predicciones no son tan precisas (Eysenck \& Eysenck, 1994).

En síntesis, cientos de estudios con el EPQ-RA muestran la replicabilidad de las dimensiones y su capacidad para predecir comportamientos a nivel social, laboral, académico, fisiológico y psicopatológico en culturas muy diferentes.

Antecedentes de estudios factoriales exploratorios con el EPQ-RA.

Hay antecedentes de análisis factorial exploratorios realizados sobre versiones abreviadas del EPQ-R (Forrest, Lewis, \& Shevlin, 2000). En dichos estudios se destaca la buena fiabilidad de todas las escalas del cuestionario, con la excepción de la escala Psicoticismo que obtiene comunalidades y pesajes factoriales más bajos que las demás. Un estudio utiliza una versión castellana del Eysenck Personality Questionnaire Revised Abbreviated (EPQ-RA, Sandín, Valiente, Chorot, Olmedo, \& Santed, 2002; Sandín, Valiente, Olmedo, Chorot, \& Santed, 2002). Es aplicada a una muestra de 263 estudiantes universitarios $(60.8 \%$ mujeres) hallándose con un estudio exploratorio la estructura factorial esperada teóricamente. Se aplicó el método de componentes principales realizándose una rotación ortogonal de la matriz original. En dicho análisis se destaca la buena fiabilidad de todas las escalas del cuestionario, con la excepción de Psicoticismo que, nuevamente, obtiene comunalidades y pesajes factoriales más bajos. Los 4 factores extraídos explicaban el $43.1 \%$ 
de la varianza total.

Un estudio realizado con estudiantes de la universidad Shippensburg, en EEUU, probó la consistencia factorial del EPQ-RA de 48 ítems (Sato, 2005). La muestra contó con un total de 309 alumnos universitarios (36.5\% hombres). La media de edad era de 19.4 años. Los coeficientes de alfa de Cronbach indicaron una buena consistencia interna de las escalas Extroversión, Neuroticismo y Mentira, con los siguientes valores .80, .78 y .73, respectivamente. La escala Psicoticismo tuvo un valor alfa aceptable pero notoriamente más bajo, .59. Se llevó a cabo un análisis de componentes principales y una rotación oblicua observándose la presencia de 4 factores que explicaron el $51.8 \%$ de la varianza.

La presente investigación tiene por objetivo adaptar el Cuestionario Revisado de Personalidad de Eysenck (versión abreviada) y explorar su comportamiento psicométrico.

\section{Método}

Participantes

La muestra, generada a través de un muestreo no probabilístico por conveniencia, estuvo constituida por 811 personas de la población general de la ciudad de Buenos Aires, de las cuales el 51.3\% eran mujeres. La edad promedio fue de 31.04 años $(D E=12.87)$. La edad máxima fue de 71 años y la edad mínima de 18 años.

\section{Instrumentos}

Se aplicó el Cuestionario EPQR-A adaptado por los autores para la población Argentina. Se trata de un cuestionario compuesto por afirmaciones acerca de la forma habitual de ser de los individuos. Cada afirmación presentada debe ser contestada de forma dicotómica (Sí-No) por el entrevistado. El cuestionario puesto a prueba al inicio de la investigación constaba de 48 ítems.

Asimismo, se aplicó un cuestionario de Datos Sociodemográficos. Se indagó edad, sexo, nivel de instrucción, ocupación, y un cuestionario clínico para conocer si cumplían criterios de inclusión (ver Procedimiento).

\section{Procedimiento}

Los participantes fueron contactados a través de conocidos y familiares de estudiantes 
de la Facultad de Psicología de la Universidad de Buenos Aires de acuerdo a criterios de inclusión. Dichos criterios fueron los siguientes: los participantes no debían ser profesionales psicólogos o estudiantes de psicología, no debían estar tomando medicación psiquiátrica ni tener diagnóstico de trastorno psicopatológico, no debían padecer convulsiones y/o ausencias repetidas, epilepsia diagnosticada, haber padecido de un episodio de coma, accidente cerebro vascular, traumatismos de cráneo con pérdida de consciencia o confusión mayor a los 30 minutos, ni consumo crónico de drogas. También se ponderó si los individuos se encontraban en buenas condiciones para realizar la tarea al momento de la evaluación, excluyéndose situaciones de fiebre, dolor, depresión o ansiedad evidentes.

\section{Tratamiento estadístico de los datos.}

Para investigar la validez de la técnica se utilizó un análisis factorial exploratorio a través del análisis de componentes principales. Se prefirió este análisis en lugar del análisis factorial confirmatorio debido a que no se buscaba probar un modelo sino conocer la estructura subyacente al conjunto de observaciones y el comportamiento de las mismas. La función de la teoría ha sido la de permitir el planteo de una serie de hipótesis respecto del número de factores que se espera encontrar y la relación entre ellos; sin embargo, el uso del EPQ-R abreviado en nuestro medio es insuficiente para especificar un modelo confirmatorio. Para extraer la cantidad de factores en el inventario se consideró el autovalor mayor a uno según el criterio Kaiser y el gráfico de sedimentación de los autovalores de Cattell (scree plot) para identificar las pendientes de la solución factorial. Se utilizó el método Varimax como método de rotación ortogonal, el cual minimiza el número de variables que tienen saturaciones altas por factor, simplificando la interpretación de los factores. Se realizaron todos estos procedimientos para la muestra completa y luego se repitieron los mismos análisis por separado para las submuestras de hombres y mujeres.

Para estimar la confiabilidad del instrumento se analizó la consistencia interna de las escalas a partir de la aplicación del alfa de Cronbach a cada una de ellas. Para establecer si los ítems de cada área eran adecuados para detectar a individuos altos y bajos en cada dimensión personológica, se llevó a cabo un estudio de discriminación de los ítems. Al tener cada ítem sólo la posibilidad de ser respondido por Si o por No, se optó por comparar ambas opciones sobre la media de cada grupo en cada escala. El test de Levene fue empleado para 
probar el supuesto de homogeneidad de las varianzas de ambos grupos. Se realizaron contrastes de medias con t de Student o su versión no paramétrica U de Mann-Withney, según se cumpliera o no el supuesto de homocedasticidad en cada comparación.

\section{Resultados}

Siguiendo el criterio propuesto por Hair, Anderson, Tatham y Black (1998) se eliminaron los ítems cuyos pesajes fueran inferiores a $.40 \mathrm{y}$ aquellos que cargaran de forma compleja para más de un solo factor. Los ítems cuyos pesajes eran inferiores a lo esperado fueron los siguientes: 9, 28, 34, 38 y 45. Sólo un ítem, el 27, cargó de forma compleja tanto para el factor Extroversión como para el factor Psicoticismo. Se decidió también extraerlo.

Luego de apartar los ítems problemáticos no se observaron alteraciones significativas en el resto de los factores. A continuación, se describen los resultados del análisis factorial exploratorio final.

En primer lugar, se realizó la medición de adecuación muestral a través del KaiserMayer-Olikin (KMO). El KMO obtenido fue de .821, indicando una adecuada interrelación entre los ítems que nos permite realizar un análisis de componentes principales de los datos. La prueba de esfericidad de Bartlett indica también valores adecuados $\left(\chi^{2}{ }_{(861)}=7204.58 ; p=\right.$ $.001)$.

Luego de aplicarse el análisis de componentes principales se obtuvieron 4 factores con autovalores superiores a la unidad. Luego de realizada la rotación de los factores, los autovalores obtenidos fueron los siguientes (entre paréntesis se indica la cantidad de varianza explicada por cada uno de ellos): Factor 1 - Neuroticismo: 4.658 (11.1\%), Factor 2 Extroversión: 3.42 (8.2\%), Factor 3 - Sinceridad: 3.00 (7.1\%), Factor 4 - Psicoticismo: 2.62 (6.3\%). Los 4 factores extraídos alcanzan a explicar el $32.65 \%$ de la varianza total.

A partir de la observación del gráfico de sedimentación se coincidió también en que los datos obtenidos ajustaban a la presencia clara de 4 factores, tal como se exhibe en la Figura 1. 


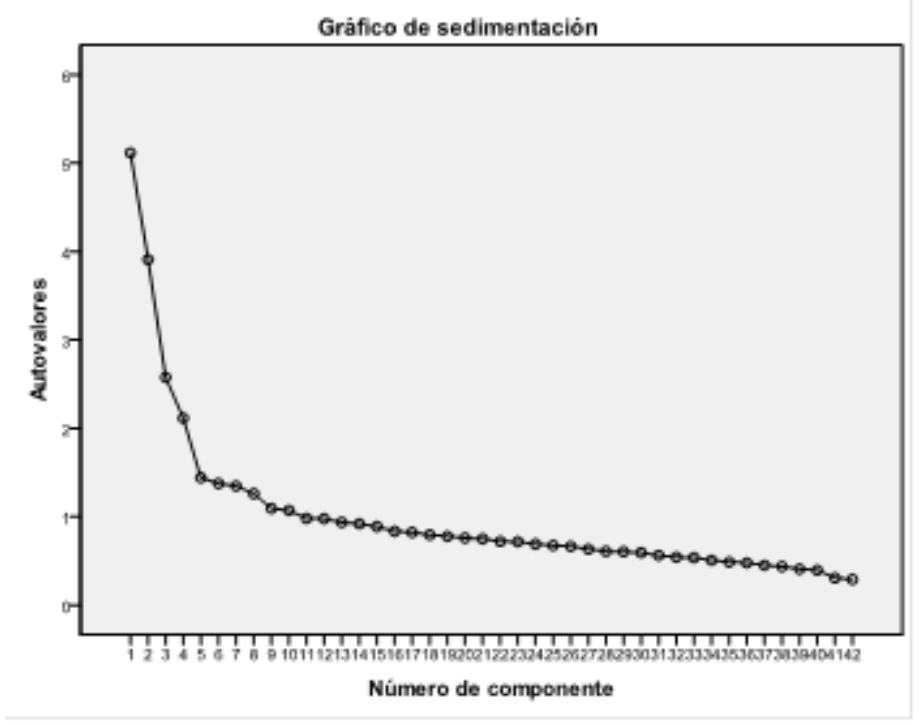

Figura 1. Gráfico de sedimentación para el EPQ-RA.

Siguiendo el criterio empleado por Tabachnick y Fidell (2001), primero se realizó una rotación ortogonal inicial y se revisaron las correlaciones entre factores. En la Tabla 1 se muestran los pesajes de cada ítem dentro de cada dimensión del modelo PEN.

Tabla 1. Pesajes de los ítems dentro de cada factor a partir del análisis factorial realizado con la muestra total de sujetos.

\begin{tabular}{|c|c|c|c|c|c|c|c|}
\hline \multicolumn{2}{|c|}{ Escala Neuroticismo } & \multicolumn{2}{|c|}{$\begin{array}{c}\text { Escala } \\
\text { Extroversión }\end{array}$} & \multicolumn{2}{|c|}{$\begin{array}{c}\text { Escala } \\
\text { Sinceridad }\end{array}$} & \multicolumn{2}{|c|}{$\begin{array}{c}\text { Escala } \\
\text { Psicoticismo }\end{array}$} \\
\hline Ítem 41 & .725 & Ítem 31 & .672 & Ítem 14 & .643 & Ítem 17 & .593 \\
\hline Ítem 02 & .693 & Ítem 47 & .659 & Ítem 36 & .603 & Ítem 23 & .570 \\
\hline Ítem 42 & .656 & Ítem 25 & .652 & Ítem 10 & .575 & Ítem 44 & .520 \\
\hline Ítem 13 & .642 & Ítem 12 & .585 & Ítem 33 & .573 & Ítem 37 & .517 \\
\hline Ítem 32 & .632 & Ítem 39 & .558 & Ítem 05 & .560 & Ítem 01 & .472 \\
\hline Ítem 20 & .612 & Ítem 22 & .544 & Ítem 21 & .524 & Ítem 40 & .446 \\
\hline Ítem 19 & .580 & Ítem 03 & .503 & Ítem 11 & .488 & Ítem 48 & .441 \\
\hline Ítem 04 & .573 & Ítem 06 & .479 & Ítem 30 & .418 & Ítem 29 & .433 \\
\hline Ítem 18 & .563 & Ítem 16 & .476 & Ítem 07 & .411 & Ítem 26 & .415 \\
\hline Ítem 24 & .546 & Ítem 46 & .445 & Ítem 43 & .401 & Ítem 15 & .414 \\
\hline Ítem 35 & .530 & & & & & & \\
\hline Ítem 08 & .402 & & & & & & \\
\hline
\end{tabular}

Nota: Rotación ortogonal- método varimax. 
La consistencia interna de las cuatro escalas fue estimada a partir de obtener un alfa de Cronbach para cada uno de los factores. Los resultados son los siguientes: para el factor Neuroticismo el valor alfa obtenido fue de .84; para el factor Extroversión, .77; para el factor Sinceridad, .71 y para el factor Psicoticismo, .66. En ninguna de las escalas se produjo que alguno de los ítems, al ser excluido, mejorara el valor del alfa. Los resultados de las escalas oscilaron entre los valores .66 a .84. Esto sugiere que dentro de cada factor los ítems muestran desde un aceptable a un muy buen grado de consistencia interna y homogeneidad.

Como otra medida de confiabilidad de los ítems que integran cada escala se miden las correlaciones totales corregidas. Para la escala Neuroticismo se observaron correlaciones adecuadas de cada ítem con la escala total, todos ellos superan .30. Ninguno de los ítems si es retirado mejora el alfa total de la escala (Tabla 2).

Tabla 2. Ítems de la escala Neuroticismo: correlaciones y alfa de Cronbach al eliminar el ítem.

\begin{tabular}{cccc}
\hline & $\begin{array}{c}\text { Correlación } \\
\text { elemento total } \\
\text { corregida }\end{array}$ & $\begin{array}{c}\text { Correlación } \\
\text { múltiple al } \\
\text { cuadrado }\end{array}$ & $\begin{array}{c}\text { Alfa de Cronbach } \\
\text { si se elimina } \\
\text { elemento }\end{array}$ \\
\hline Ítem 02 & .613 & .502 & .821 \\
Ítem 04 & .477 & .279 & .832 \\
Ítem 08 & .323 & .195 & .843 \\
Ítem 13 & .577 & .360 & .824 \\
Ítem 18 & .438 & .225 & .835 \\
Ítem 19 & .485 & .354 & .831 \\
Ítem 20 & .495 & .283 & .831 \\
Ítem 24 & .456 & .336 & .833 \\
Ítem 32 & .538 & .343 & .827 \\
Ítem 35 & .453 & .250 & .834 \\
Ítem 41 & .651 & .538 & .818 \\
Ítem 42 & .569 & .384 & .825 \\
\hline
\end{tabular}

Nota: Los valores representan las correlaciones totales corregidas, correlaciones múltiples al cuadrado y las modificaciones en el alfa de Cronbach si se elimina el ítem de la escala total.

Para la escala Extroversión se observaron correlaciones adecuadas de cada ítem con la escala total, todos ellos superan .30. Ninguno de los ítems si es retirado mejora el alfa total de la escala (Tabla 3). 
Tabla 3. Ítems de la escala Extroversión: correlaciones y alfa de Cronbach al eliminar el ítem.

\begin{tabular}{cccc}
\hline Ítem & $\begin{array}{c}\text { Correlación } \\
\text { elemento total } \\
\text { corregida }\end{array}$ & $\begin{array}{c}\text { Correlación } \\
\text { múltiple al } \\
\text { cuadrado }\end{array}$ & $\begin{array}{c}\text { Alfa de } \\
\text { Cronbach si se } \\
\text { elimina elemento }\end{array}$ \\
\hline Ítem 03 & .341 & .157 & .760 \\
Ítem 06 & .387 & .202 & .754 \\
Ítem 12 & .459 & .254 & .744 \\
Ítem 16 & .368 & .166 & .756 \\
Ítem 22 & .410 & .175 & .752 \\
Ítem 25 & .504 & .297 & .738 \\
Ítem 31 & .555 & .333 & .731 \\
Ítem 39 & .448 & .227 & .746 \\
Ítem 46 & .337 & .147 & .762 \\
Ítem 47 & .538 & .326 & .735
\end{tabular}

Nota: Los valores representan las correlaciones totales corregidas, correlaciones múltiples al cuadrado y las modificaciones en el alfa de Cronbach si se elimina el ítem de la escala total.

Para la escala Psicoticismo se observaron correlaciones adecuadas de cada ítem con la escala total, la mayoría de ellos superan el criterio .30. Sin embargo, los ítems 26, 29 y 48 obtienen correlaciones inferiores a este parámetro. Ninguno de los ítems si es retirado mejora el alfa total de la escala (Tabla 4).

Tabla 4. Ítems de la escala Psicoticismo: correlaciones y alfa de Cronbach al eliminar el ítem.

\begin{tabular}{cccc}
\hline Ítem & $\begin{array}{c}\text { Correlación } \\
\text { elemento } \\
\text { total corregida }\end{array}$ & $\begin{array}{c}\text { Correlación } \\
\text { múltiple al } \\
\text { cuadrado }\end{array}$ & $\begin{array}{c}\text { Alfa de } \\
\text { Cronbach si se } \\
\text { elimina elemento }\end{array}$ \\
\hline Ítem 01 & .367 & .444 & .632 \\
Ítem 15 & .273 & .082 & .650 \\
Ítem 17 & .440 & .324 & .613 \\
Ítem 23 & .389 & .224 & .625 \\
Ítem 26 & .257 & .076 & .652 \\
Ítem 29 & .232 & .068 & .656 \\
Ítem 37 & .351 & .262 & .636 \\
Ítem 40 & .328 & .440 & .639 \\
Ítem 44 & .348 & .233 & .635 \\
Ítem 48 & .266 & .227 & .652
\end{tabular}

Nota: Los valores representan las correlaciones totales corregidas, correlaciones múltiples al cuadrado y las modificaciones en el alfa de Cronbach si se elimina el ítem de la escala total. 
Para la escala Sinceridad se observaron correlaciones adecuadas de cada ítem con la escala total, la mayoría de ellos superan el criterio .30. Sin embargo, los ítems 07 y 43 obtienen correlaciones inferiores a este parámetro. Ninguno de los ítems si es retirado mejora el alfa total de la escala (Tabla 5).

Tabla 5. Ítems de la escala Sinceridad: correlaciones y alfa de Cronbach al eliminar el ítem.

\begin{tabular}{cccc}
\hline Ítem & $\begin{array}{c}\text { Correlación } \\
\text { elemento total } \\
\text { corregida }\end{array}$ & $\begin{array}{c}\text { Correlación } \\
\text { múltiple al } \\
\text { cuadrado }\end{array}$ & $\begin{array}{c}\text { Alfa de Cronbach } \\
\text { si se elimina } \\
\text { elemento }\end{array}$ \\
\hline Ítem 05 & .389 & .186 & .689 \\
Ítem 07 & .297 & .125 & .704 \\
Ítem 10 & .427 & .216 & .683 \\
Ítem 11 & .361 & .151 & .693 \\
Ítem 14 & .449 & .250 & .678 \\
Ítem 21 & .379 & .160 & .690 \\
Ítem 30 & .300 & .122 & .704 \\
Ítem 33 & .403 & .187 & .686 \\
Ítem 36 & .453 & .242 & .680 \\
Ítem 43 & .278 & .112 & .707
\end{tabular}

Nota: Los valores representan las correlaciones totales corregidas, correlaciones múltiples al cuadrado y las modificaciones en el alfa de Cronbach si se elimina el ítem de la escala total.

El análisis de discriminación de los ítems indica que los ítems distinguen de manera confiable entre bajas y altas puntuaciones en cada una de las tres dimensiones de personalidad y la escala de sinceridad (Tablas 6, 7, 8 y 9). 
Tabla 6. Discriminación de los ítems de la escala de Neuroticismo.

\begin{tabular}{ccccc}
\hline Ítem & Levene & p valor & Prueba utilizada & p valor \\
\hline 02 & $\mathrm{~F}=19.589$ & $p=.000$ & $\mathrm{Z}=-18.755$ & $p=.000$ \\
04 & $\mathrm{~F}=.347$ & $p=.556$ & $\mathrm{t}_{(707)}=-18.635$ & $p=.000$ \\
08 & $\mathrm{~F}=1.505$ & $p=.220$ & $\mathrm{t}_{(707)}=-12.959$ & $p=.000$ \\
13 & $\mathrm{~F}=8.729$ & $p=.003$ & $\mathrm{Z}=-17.669$ & $p=.000$ \\
18 & $\mathrm{~F}=.079$ & $p=.778$ & $\mathrm{t}_{(707)}=-17.391$ & $p=.000$ \\
19 & $\mathrm{~F}=6.666$ & $p=.010$ & $\mathrm{Z}=-15.651$ & $p=.000$ \\
20 & $\mathrm{~F}=16.348$ & $p=.000$ & $\mathrm{Z}=-15.148$ & $p=.000$ \\
24 & $\mathrm{~F}=1.764$ & $p=.185$ & $\mathrm{t}_{(707)}=-17.988$ & $p=.000$ \\
32 & $\mathrm{~F}=1.670$ & $p=.197$ & $\mathrm{t}_{(707)}=-21.440$ & $p=.000$ \\
35 & $\mathrm{~F}=.207$ & $p=.649$ & $\mathrm{t}_{(707)}=-18.022$ & $p=.000$ \\
41 & $\mathrm{~F}=11.856$ & $p=.001$ & $\mathrm{Z}=-19.449$ & $p=.000$ \\
42 & $\mathrm{~F}=3.703$ & $p=.055$ & $\mathrm{t}_{(707)}=-23.465$ & $p=.000$
\end{tabular}

Nota: Se indican las pruebas utilizadas cuando se cumple el supuesto de homogeneidad de las varianzas (test de Levene) y cuando este supuesto no puede asumirse. Si la homocedasticidad puede probarse o no, se utiliza el test $t$ de Student ( $\mathrm{t}$ ) o la U de Mann-Whitney (Z), respectivamente.

Tabla 7. Discriminación de los ítems de la escala de Extroversión.

\begin{tabular}{ccccc}
\hline Ítem & Levene & p valor & Prueba utilizada & p valor \\
\hline 03 & $\mathrm{~F}=8.299$ & $p=.004$ & $\mathrm{Z}=-12.313$ & $p=.000$ \\
06 & $\mathrm{~F}=.722$ & $p=.369$ & $\mathrm{t}_{(710)}=-13.708$ & $p=.000$ \\
12 & $\mathrm{~F}=6.529$ & $p=.011$ & $\mathrm{Z}=-14.047$ & $p=.000$ \\
16 & $\mathrm{~F}=3.537$ & $p=.060$ & $\mathrm{t}_{(710)}=-15.592$ & $p=.000$ \\
22 & $\mathrm{~F}=21.564$ & $p=.000$ & $\mathrm{Z}=-15.176$ & $p=.000$ \\
25 & $\mathrm{~F}=17.475$ & $p=.000$ & $\mathrm{Z}=-15.807$ & $p=.000$ \\
31 & $\mathrm{~F}=7.238$ & $p=.007$ & $\mathrm{Z}=-16.327$ & $p=.000$ \\
39 & $\mathrm{~F}=22.264$ & $p=.000$ & $\mathrm{Z}=-17.028$ & $p=.000$ \\
46 & $\mathrm{~F}=42.911$ & $p=.000$ & $\mathrm{Z}=-13.865$ & $p=.000$ \\
47 & $\mathrm{~F}=.170$ & $p=.680$ & $\mathrm{t}_{(710)}=-22.356$ & $p=.000$
\end{tabular}

Nota: Se indican las pruebas utilizadas cuando se cumple el supuesto de homogeneidad de las varianzas (test de Levene) y cuando este supuesto no puede asumirse. Si la homocedasticidad puede probarse o no, se utiliza el test t de Student ( $\mathrm{t}$ ) o la U de Mann-Whitney (Z), respectivamente. 
Tabla 8. Discriminación de los ítems de la escala de Psicoticismo.

\begin{tabular}{ccccc}
\hline Ítem & Levene & p valor & Prueba utilizada & p valor \\
\hline 01 & $\mathrm{~F}=2.315$ & $p=.129$ & $\mathrm{t}_{(709)}=-13.782$ & $p=.000$ \\
15 & $\mathrm{~F}=.550$ & $p=.459$ & $\mathrm{t}_{(709)}=-12.904$ & $p=.000$ \\
17 & $\mathrm{~F}=5.047$ & $p=.025$ & $\mathrm{Z}=-16.775$ & $p=.000$ \\
23 & $\mathrm{~F}=.126$ & $p=.723$ & $\mathrm{t}_{(709)}=-16.844$ & $p=.000$ \\
26 & $\mathrm{~F}=.001$ & $p=.977$ & $\mathrm{t}_{(709)}=-9.929$ & $p=.000$ \\
29 & $\mathrm{~F}=1.182$ & $p=.277$ & $\mathrm{t}_{(709)}=-11.782$ & $p=.000$ \\
37 & $\mathrm{~F}=.398$ & $p=.528$ & $\mathrm{t}_{(709)}=-14.456$ & $p=.000$ \\
40 & $\mathrm{~F}=.126$ & $p=.722$ & $\mathrm{t}_{(709)}=-13.735$ & $p=.000$ \\
44 & $\mathrm{~F}=.8 .285$ & $p=.004$ & $\mathrm{Z}=-14.359$ & $p=.000$ \\
48 & $\mathrm{~F}=1.389$ & $p=.239$ & $\mathrm{t}_{(709)}=-12.472$ & $p=.000$
\end{tabular}

Nota: Se indican las pruebas utilizadas cuando se cumple el supuesto de homogeneidad de las varianzas (test de Levene) y cuando este supuesto no puede asumirse. Si la homocedasticidad puede probarse o no, se utiliza el test $t$ de Student ( $\mathrm{t}$ ) o la U de Mann-Whitney (Z), respectivamente.

Tabla 9. Discriminación de los ítems de la escala de Sinceridad.

\begin{tabular}{ccccc}
\hline Ítem & Levene & p valor & Prueba utilizada & p valor \\
\hline 05 & $\mathrm{~F}=2.406$ & $p=.121$ & $\mathrm{t}_{(713)}=-17.332$ & $p=.000$ \\
07 & $\mathrm{~F}=2.130$ & $p=.145$ & $\mathrm{t}_{(713)}=-13.509$ & $p=.000$ \\
10 & $\mathrm{~F}=7.805$ & $p=.005$ & $\mathrm{Z}=-3.826$ & $p=.000$ \\
11 & $\mathrm{~F}=.409$ & $p=.523$ & $\mathrm{t}_{(713)}=-15.414$ & $p=.000$ \\
14 & $\mathrm{~F}=2.447$ & $p=.118$ & $\mathrm{t}_{(713)}=-19.946$ & $p=.000$ \\
21 & $\mathrm{~F}=3.606$ & $p=.058$ & $\mathrm{t}_{(713)}=-16.248$ & $p=.000$ \\
30 & $\mathrm{~F}=8.452$ & $p=.004$ & $\mathrm{Z}=-1.997$ & $p=.046$ \\
33 & $\mathrm{~F}=1.654$ & $p=.199$ & $\mathrm{t}_{(713)}=-17.980$ & $p=.000$ \\
36 & $\mathrm{~F}=20.053$ & $p=.000$ & $\mathrm{Z}=-4.568$ & $p=.000$ \\
43 & $\mathrm{~F}=.078$ & $p=.780$ & $\mathrm{t}_{(713)}=-12.599$ & $p=.000$
\end{tabular}

Nota: Se indican las pruebas utilizadas cuando se cumple el supuesto de homogeneidad de las varianzas (test de Levene) y cuando este supuesto no puede asumirse. Si la homocedasticidad puede probarse o no, se utiliza el test t de Student (t) o la U de Mann-Whitney (Z), respectivamente.

Se llevó a cabo el análisis factorial de forma independiente para hombres y mujeres. El análisis factorial exploratorio para mujeres arrojó resultados similares, sin necesidad de extraer nuevos ítems y sin que surgieran comportamientos complejos en los mismos.

Volvió a realizarse la medición de adecuación muestral para el subgrupo de las mujeres. El KMO obtenido fue de .799, indicando también una adecuada interrelación entre 
los ítems que nos permite realizar un análisis de componentes principales de los datos. La prueba de esfericidad de Bartlett volvió a indicar valores adecuados $\left(\chi^{2}{ }_{(861)}=4042.70, p=\right.$ .001). Luego de aplicarse el análisis de componentes principales a la muestra de mujeres se obtuvieron los mismos 4 factores con autovalores superiores a la unidad. Luego de realizada la rotación de los factores, los autovalores obtenidos fueron los siguientes (entre paréntesis se indica la cantidad de varianza explicada por cada uno de ellos): Factor 1 - Neuroticismo: 4.778 (11.4\%), Factor 2 - Extroversión: 3.328 (7.9\%), Factor 3 - Sinceridad: 3.254 (7.7\%), Factor 4 - Psicoticismo 2.662 (6.3\%). Los 4 factores extraídos alcanzan a explicar el 33.39\% de la varianza total. En la Tabla 10 pueden observarse los pesajes obtenidos dentro de cada factor por cada uno de los ítems al realizarse el análisis solo para el caso de las mujeres.

Tabla 10. Análisis de componentes principales realizada solo a la muestra de mujeres.

\begin{tabular}{|c|c|c|c|c|c|c|c|}
\hline \multicolumn{2}{|c|}{ Escala Neuroticismo } & \multicolumn{2}{|c|}{ Escala Extroversión } & \multicolumn{2}{|c|}{ Escala Sinceridad } & \multicolumn{2}{|c|}{ Escala Psicoticismo } \\
\hline Ítem 41 & .724 & Ítem 47 & .708 & Ítem 33 & .624 & Ítem 17 & .603 \\
\hline Ítem 02 & .698 & Ítem 25 & .671 & Ítem 14 & .611 & Ítem 44 & .535 \\
\hline Ítem 42 & .682 & Ítem 31 & .664 & Ítem 36 & .585 & Ítem 01 & .515 \\
\hline Ítem 13 & .634 & Ítem 39 & .569 & Ítem 05 & .571 & Ítem 40 & .497 \\
\hline Ítem 04 & .633 & Ítem 12 & .566 & Ítem 10 & .564 & Ítem 37 & .496 \\
\hline Ítem 32 & .622 & Ítem 22 & .503 & Ítem 21 & .535 & Ítem 15 & .479 \\
\hline Ítem 20 & .621 & Ítem 16 & .461 & Ítem 07 & .514 & Ítem 23 & .466 \\
\hline Ítem 35 & .569 & Ítem 06 & .427 & Ítem 11 & .503 & Ítem 26 & .449 \\
\hline Ítem 24 & .556 & Ítem 46 & .404 & Ítem 43 & .470 & Ítem 29 & .443 \\
\hline Ítem 19 & .553 & Ítem 03 & .308 & Ítem 30 & .405 & Ítem 48 & .389 \\
\hline Ítem 18 & .546 & & & & & & \\
\hline Ítem 08 & .406 & & & & & & \\
\hline
\end{tabular}

Nota: Los valores representan los pesajes de los ítems dentro de cada factor. Rotación ortogonal - método varimax.

El análisis factorial exploratorio para la muestra de hombres indicó nuevamente resultados similares, sin necesidad de extraer nuevos ítems y no surgieron comportamientos complejos en los mismos.

La medición de adecuación muestral para el subgrupo de hombres indica un KMO de .773, siendo esta, también una adecuada interrelación entre los ítems que nos permite realizar un análisis de componentes principales de los datos. La prueba de esfericidad de Bartlett 
vuelve a indicar valores adecuados $\left(\chi^{2}{ }_{(861)}=3881.88, p=.001\right)$.

Luego de aplicarse el análisis de componentes principales a la muestra de hombres se volvieron a obtener los mismos 4 factores con autovalores superiores a la unidad. Luego de realizada la rotación ortogonal de los factores, los autovalores obtenidos fueron los siguientes (entre paréntesis se indica la cantidad de varianza explicada por cada uno de ellos). Factor 1 Neuroticismo: 4.537 (10.8\%), Factor 2 - Extroversión: 3.612 (8.6\%), Factor - 3 Sinceridad: 2.778 (6.6), factor 4 - Psicoticismo: 2.711 (6.4\%). Los 4 factores extraídos alcanzan a explicar el $32.47 \%$ de la varianza total. En la Tabla 11 pueden observarse los pesajes obtenidos dentro de cada factor por cada uno de los ítems al realizarse el análisis sólo para el caso de los hombres.

Tabla 11. Análisis de componentes principales realizada solo a la muestra de hombres.

\begin{tabular}{|c|c|c|c|c|c|c|c|}
\hline \multicolumn{2}{|c|}{ Escala Neuroticismo } & \multicolumn{2}{|c|}{ Escala Extroversión } & \multicolumn{2}{|c|}{ Escala Sinceridad } & \multicolumn{2}{|c|}{ Escala Psicoticismo } \\
\hline Ítem 41 & .717 & Ítem 31 & .669 & Ítem 14 & .663 & Ítem 23 & .645 \\
\hline Ítem 02 & .673 & Ítem 25 & .620 & Ítem 36 & .606 & Ítem 17 & .573 \\
\hline Ítem 13 & .660 & Ítem 12 & .613 & Ítem 10 & .581 & Ítem 37 & .514 \\
\hline Ítem 19 & .644 & Ítem 47 & .603 & Ítem 05 & .568 & Ítem 44 & .506 \\
\hline Ítem 32 & .623 & Ítem 03 & .594 & Ítem 11 & .486 & Ítem 48 & .461 \\
\hline Ítem 42 & .597 & Ítem 22 & .587 & Ítem 21 & .470 & Ítem 01 & .444 \\
\hline Ítem 20 & .584 & Ítem 39 & .554 & Ítem 33 & .417 & Ítem 29 & .424 \\
\hline Ítem 24 & .579 & Ítem 06 & .519 & Ítem 30 & .340 & Ítem 40 & .419 \\
\hline Ítem 18 & .552 & Ítem 46 & .466 & Ítem 07 & .338 & Ítem 26 & .346 \\
\hline Ítem 35 & .496 & Ítem 16 & .463 & Ítem 43 & .325 & Ítem 15 & .345 \\
\hline Ítem 04 & .473 & & & & & & \\
\hline Ítem 08 & .417 & & & & & & \\
\hline
\end{tabular}

Nota: Los valores representan los pesajes de los ítems dentro de cada factor. Rotación ortogonal - método varimax.

\section{Conclusiones}

En el presente trabajo se exponen los resultados de la adaptación local del EPQ-RA (Eysenck, \& Eysenck, 2001). Se extrajeron los tres factores de personalidad hipotetizados por el modelo Psicoticismo-Extroversión-Neuroticismo (PEN) y uno de sinceridad. Se realizaron los mismos análisis por separado para las submuestras de hombres y mujeres con los mismos 
buenos resultados en términos psicométricos. Para estimar la confiabilidad del instrumento se aplicó el alfa de Cronbach, observándose adecuados índices de consistencia interna para cada subescala. De este modo, se ha obtenido la versión local del EPQ-RA que consta de 42 ítems distribuidos en tres factores robustos que se corresponden con las tres dimensiones de personalidad más prolíficas de la historia de la Psicología, y un factor de sinceridad igualmente válido y consistente que permite conocer la veracidad de las respuestas del sujeto o una tendencia al conformismo social.

Las investigaciones centradas en dimensiones básicas de personalidad son posibles si se cuenta con instrumentos adecuadamente adaptados. A partir de allí, es posible pensar en los rasgos y comportamientos vinculados a la unidad personológica básica, es decir, las dimensiones. La adaptación presentada no sólo tiene un fin instrumental sino que permite avanzar en la discusión aún vigente respecto de cuántas y cuáles son las dimensiones básicas.

En el futuro se indagarán los factores resultantes con criterios externos y se ampliará la muestra, pues la misma no es representativa y, por lo tanto, los resultados no son generalizables.

Es probable que se hayan encontrado algunos desajustes entre la teoría y los datos empíricos dado que no todos las observaciones propuestas por Eysenck se comportan de la manera esperable (por ejemplo, ítems que no responden a ninguno de los cuatro factores e ítems cuyo pesaje es mayor en un factor diferente del hipotetizado inicialmente) y es probable que en algunos casos sea necesaria una formulación más compleja. Los mayores desacuerdos se encuentran en el Psicoticismo. Mientras que para Eysenck esta es la tercera y última dimensión básica, para otros autores (por ejemplo, Costa \& McCrae, 1992) existen más de tres factores. Sin embargo, la regla general en ciencia es que una teoría se descarta normalmente solo cuando otra superior la sustituye y, hasta ahora, esto no ha ocurrido. Es necesario acumular conocimiento y avanzar en la misma dirección. La proliferación de teorías, rasgos e instrumentos retrasa la posibilidad de acumulación de conocimiento.

Se necesita contrastar predicciones a partir del EPQ-RA a nivel fisiológico, de condicionamiento y de conducta social. El análisis factorial confirmatorio también puede arrojar algún resultado interesante, por lo que se continuará indagando la validez y las posibles aplicaciones de este instrumento en nuestro medio. 


\section{Referencias}

Brody, N. \& Ehrlichman, H. (2000). Psicología de la Personalidad. Madrid: Prentice Hall.

Costa, P. T., Jr., \& McCrae, R. R. (1992). NEO-PI-R Professional Manual. PAR, Florida.

Eysenck, H.J. (1952). The scientific study of personality. London: Routledge \& Kegan Paul.

Eysenck, H.J. (1959). Manual of the Maudsley Personality Inventory. London: University of London Press.

Eysenck, H. J. (1967). The biological bases of Personality. Springfield, I.L.: Charles C. Thomas.

Eysenck, H. J. (1987). Personalidad y diferencias Individuales. Madrid. Ediciones Pirámide, S. A.

Eysenck, H. J. (1990). Genetic and environmental contributions to individual differences: the three major dimensions of personality. Journal of Personality, 58, 245-261.

Eysenck, H.J., \& Eysenck, S.B.G. (1975). Manual of the Eysenck Personality Questionnaire.London: Hodder \& Stoughton.

Eysenck, H.J., \& Eysenck, S.B.G. (1983). EPQ:Cuestionario de personalidad. Madrid: Técnicos Especialistas Asociados.

Eysenck, H. J., \& Eysenck, S. B. G. (1994). Manual of the Eysenck Personality Questionnaire. California: EdITS/Educational and Industrial Testing Service.

Eysenck, H. J., \& Eysenck, S. B. G. (2001). Cuestionario revisado de Personalidad de Eysenck. Manual. Madrid: TEA Ediciones,

Eysenck, S.B.G., \& Eysenck, H.J. (1964). Manual of the Eysenck Personality Inventory. London: University of London Press.

Forrest, S., Lewis, C.A., \& Shevlin, M. (2000). Examiningm the factor structure and differential functioning of the Eysenck Personality Questionnaire RevisedAbbreviated. Personality and Individual Differences, 29, 579-588.

Gutiérrez-Maldonado, J. (1999) (ed.): Psicología hoy. CADUP, estudios: Tortosa. 7-11. CL ISBN: 84-8416-640-6

Hair, J., Anderson, R., Tatham, R., \& Black, W. (1998). Multivariable data analysis. NJ: Prentice Hall.

LeBlanc, J., \& Ducharme, M.B. (2005). Influence of personality traits on plasma levels of cortisol and cholesterol. Physiology \& Behavior, 84, 677-680.

Pelechano-Barberá, V. (2000 ). Psicología Sistemática de la Personalidad. Barcelona: Ariel 
Psicología.

Sandín, B., Valiente, R. M., Chorot, P., Olmedo, M., \& Santed, M. A. (2002). Versión española del cuestionario EPQR-Abreviado (EPQR-A) (I): Análisis exploratorio de la estructura factorial. Revista de Psicopatología y Psicología Clínica, 7(3), 195-205.

Sandín, B., Valiente, R. M., Olmedo, M., Chorot, P., \& Santed, M. A. (2002). Versión española del cuestionario EPQR-Abreviado (EPQR-A) (II): Replicación factorial, fiabilidad y validez. Revista de Psicopatología y Psicología Clínica, 7(3), 207-216.

Sato, T. (2005). The Eysenck Personality Questionnaire Brief Version: Factor Structure and Reliability. The Journal of Psychology, 2005, 139(6), 545-552

Schmidtke, J. I., \& Heller, W. (2004). Personality, affect and EEG: predicting patterns of regional brain activity related to extraversion and neuroticism. Personality and Individual Differences, 36, 717-732.

Schmidt, V., Firpo, L., Vion, D., De Costa Oliván, M. E., Casella, L., Cuenya, L, Blum, G.D., \& Pedrón, V. (2009). Modelo psicobiológico de personalidad de Eysenck: una historia proyectada hacia el futuro. Revista Internacional de Psicología, 11(2), 3-27.

Squillace, M. (2009). Estrategias multimétodo para la evaluación de la impulsividad. Memorias del IV Congreso de Psicología de la Universidad de Mar del Plata.

Squillace, M., Martin, L., Gianotti, J., Pedrón, V., \& Yorio A. (2008). Diferencias individuales en adicciones: perfiles psicosociales asociados al consumo de cocaína. Investigaciones en Psicología, 13(3), 73-85.

Squillace, M., Picón Janeiro, J., \& Schmidt, V. (2011). El concepto de Impulsividad y su ubicación en las teorías psicobiológicas de la personalidad. Revista Neuropsicología Latinoamericana 3(1), 8-18.

Stelmack, R.M. (1990). Biological Bases of Extraversion Psychophysiological Evidence, Journal of Personality, 58, 298-311.

Tabachnick, B. \& Fidell, L. (2001). Using multivaraite statistics. Nueva York: Harper \& Row.

Wilson, E. L. (1990) Personality Characteristics of Adult Children of Alcoholics, Journal of Humanistic Education and Development, 28, 155-175

Zuckerman, M. (1992). What is a basic factor and which factors are basic? Turtles all the way down. Personality and Individual Differences, 13, 675-681. 